\title{
$\checkmark$ Research Square

\section{Honey and Nigella Sativa in the Prophylaxis of COVID-19; A Randomized Controlled Trial}

Sohaib Ashraf ( $\square$ sohaib-ashraf@outlook.com )

Shaikh Zayed Medical Complex https://orcid.org/0000-0003-3127-3557

\section{Shoaib Ashraf}

Riphah International University

\section{Rutaba Akmal}

Sahara Hospital

\section{Moneeb Ashraf}

King Edward Medical College: King Edward Medical University

\section{Larab Kalsoom}

Services Institute of Medical Sciences

\section{Aadil Maqsood}

University of Toledo - Health Science Campus

\section{Muhammad Ahmad Imran}

Shaikh Zayed Postgraduate Medical Institute

\section{Iqra Farooq}

children hospital

\section{Sidra Ashraf}

University of Veterinary and Animal Sciences

\section{Uzma Nasim Siddique}

Shaikh Zayed Postgraduate Medical Institute

\section{Muhammad Ghufran}

ESACHE

\section{Muhammad Kiwan Akram}

University of Veterinary and Animal Sciences

\section{Nighat Majeed}

Services Institute of Medical Sciences

\section{Sundas Rafique}

Mayo Hospital

\section{Zaighum Habib}

Shaikh Zayed Postgraduate Medical Institute

\section{Muhammad Sarmad Shahab}

Allied Hospital, The University of Faisalabad

\section{Adeen Akmal}


University of Veterinary and Animal Sciences

\section{Zeeshan Shaukat}

Shaikh Zayed Postgraduate Medical Institute

\section{Zain ul Abdin}

Shaikh Zayed Postgraduate Medical Institute

\section{Ayesha Khaqan}

Shaikh Zayed Postgraduate Medical Institute

\section{Shahroze Arshad}

Shaikh Zayed Postgraduate Medical Institute

\section{Muhammad Abdulrehman Virk}

Shaikh Zayed Postgraduate Medical Institute

\section{Mehak Gul}

Shaikh Zayed Postgraduate Medical Institute

\section{Abeer Bin Awais}

Shaikh Zayed Postgraduate Medical Institute

\section{Muhammad Hassan}

Shaikh Zayed Postgraduate Medical Institute

\section{Noman Khalid}

Shaikh Zayed Postgraduate Medical Institute

\section{Qurrat-ul-Ain Iqbal}

Shaikh Zayed Postgraduate Medical Institute

\section{Tausif Ahmad}

Shaikh Zayed Postgraduate Medical Institute

\section{Muaaz Akram}

Sheikh Zayed Post Graduate Medical Institute

Ameer Muhammad

Shaikh Zayed Postgraduate Medical Institute

\section{Musa Khalil}

Shaikh Zayed Postgraduate Medical Institute

\section{Aneeq Aslam}

Shaikh Zayed Postgraduate Medical Institute

\section{Muhammad Umer}

Shaikh Zayed Postgraduate Medical Institute

\section{Syed Sami Hussain Sherazi}

University of Veterinary and Animal Sciences

\section{Zartasha Safdar}

University of Veterinary and Animal Sciences

\section{Sohail Ahmad}

University of Veterinary and Animal Sciences 


\section{Muhammad Bilal}

McGill University

\section{Muhammad Nauman Zahid}

University of Bahrain

\section{Abdulrahman E Koshak}

King Abdulaziz Medical City - Jeddah

\section{Abubakar Hilal}

Shaikh Zayed Postgraduate Medical Institute

\section{Ahmad Azam Malik}

King Abdulaziz Medical City - Jeddah

\section{Usman lqbal}

Taipei Medical University

\section{Atif Amin Baig}

Sultan Zainal Abidin Religious College: Universiti Sultan Zainal Abidin

\section{Yaser Masuod Alahmadi}

Taibah University

\section{Ayesha Humayun}

Shaikh Zayed Postgraduate Medical Institute

\section{Amber Malik}

Shaikh Zayed Postgraduate Medical Institute

\section{Ali Ahmad}

University of Montreal Hospital Centre Research Centre: Centre Hospitalier de l'Universite de Montreal Centre de Recherche

\section{Muhammad Ashraf}

University of Veterinary and Animal Sciences

\section{Qazi Abdul Saboor}

Shaikh Zayed Postgraduate Medical Institute

\section{Mateen Izhar}

Shaikh Zayed Postgraduate Medical Institute

\section{Doctors Lounge Consortium}

Shaikh Zayed Postgraduate Medical Institute

\section{Research Article}

Keywords: Honey, Nigella Sativa, Prophetic Medicine, Pakistan, COVID-19, Randomised controlled trial, Protocol

Posted Date: August 9th, 2021

DOI: https://doi.org/10.21203/rs.3.rs-757643/v1 
License: (c) (i) This work is licensed under a Creative Commons Attribution 4.0 International License. Read Full License 


\section{Abstract}

\section{Objectives}

Considering the therapeutic potential of honey and Nigella sativa (HNS) in coronavirus disease 2019 (COVID-19) patients, the objective of the study is defined to evaluate the prophylactic role of HNS.

\section{Trial design}

The study is a randomized, placebo-controlled, adaptive clinical trial with parallel group design, superiority framework with an allocation ratio of 1:1 among experimental (HNS) and placebo group. An interim analysis will be done when half of the patients have been recruited to evaluate the need to adapt sample size, efficacy, and futility of the trial.

\section{Participants}

All asymptomatic patients with hospital or community based COVID-19 exposure will be screened if they have had 4 days exposure to a confirmed case. Non-pregnant adults with significant exposure level will be enrolled in the study

- High-risk exposure ( $<6$ feet distance for $>10$ min without face protection)

- Moderate exposure ( $<6$ feet distance for $>10$ min with face protection)

Subjects with acute or chronic infection, COVID-19 vaccinated, and allergy to HNS will be excluded from the study.

Recruitment will be done at Shaikh Zayed Post-Graduate Medical Institute, Ali Clinic and Doctors Lounge in Lahore (Pakistan).

\section{Intervention and comparator}

In this clinical study, patients will receive either raw natural honey $(0.5 \mathrm{~g})$ and encapsulated organic Nigella sativa seeds $(40 \mathrm{mg}$ ) per kg body weight per day or empty capsule with and $30 \mathrm{ml}$ of $5 \%$ dextrose water as a placebo for 14 days. Both the natural products will be certified for standardization by Government College University (Botany department). Furthermore, each patient will be given standard care therapy according to version 3.0 of the COVID-19 clinical management guidelines by the Ministry of National Health Services of Pakistan.

\section{Main outcomes}

Primary outcome will be Incidence of COVID-19 cases within 14 days of randomisation. Secondary endpoints include incidence of COVID-19-related symptoms, hospitalizations, and deaths along with the severity of COVID-19-related symptoms till $14^{\text {th }}$ day of randomization. 


\section{Randomisation}

Participants will be randomized into experimental and control groups (1:1 allocation ratio) via the lottery method. There will be stratification based on high risk and moderate risk exposure.

\section{Blinding (masking)}

Quadruple blinding will be ensured for the participants, care providers and outcome accessors. Data analysts will also be blinded to avoid conflict of interest. Site principal investigator will be responsible for ensuring masking.

\section{Numbers to be randomised (sample size)}

1000 participants will be enrolled in the study with 1:1 allocation.

\section{Trial Status}

The final protocol version 1.4 was approved by institutional review board of Shaikh Zayed Post-Graduate Medical Complex on February 15, 2021. The trial recruitment was started on March 05, 2021, with a trial completion date of February 15, 2022.

\section{Trial registration}

Clinical trial was registered on February 23, 2021, www.clinicaltrials.gov with registration ID NCT04767087.

\section{Full Protocol}

The full protocol is attached as an additional file, accessible from the Trials website (Additional file 1). With the intention of expediting dissemination of this trial, the conventional formatting has been eliminated; this Letter serves as a summary of the key elements of the full protocol. The study protocol has been reported in accordance with the Standard Protocol Items: Recommendations for Clinical Interventional Trials (SPIRIT) guidelines.

\section{Declarations}

\section{Ethics approval and consent to participate}

The trial was approved by institutional ethical review board of Shaikh Zayed Post-Graduate Medical Complex (IRB ID \# SZMC/IRB/Internal/273/2021) on February 15, 2021. Authors certify that the study has received ethical approval from the appropriate ethical committee as stated above. Before enrolment, all partakers will be fully informed of the study and asked to sign the consent form to be eligible for randomization and participation. 


\section{Consent for publication}

Not applicable

\section{Availability of data and materials}

All required data will be posted along with the trial results and final trail dataset requests can be made to Dr. Sohaib Ashraf which could be available from the author on reasonable appeal subject to data protection regulations. (Twitter: SohaibAshrafMD, email address: sohaib@skzmdc.edu.pk, Mobile Number: +92 3334474523)

\section{Competing interests}

The authors declare that they have no competing interests.

\section{Funding}

Funding by Smile Welfare Organization (SWO) - a non-profitable organization. The funder played no role in the design of the study, in the collection, analysis, and interpretation of data, and in writing the manuscript.

\section{Authors' contributions}

SA, ShA, MA, AM, SA, MAI, LK, UNS, NM, IF, RK, and MG contributed equally to this paper and share joint first authorship. SA, ShA, MA, AM, and AA are joint corresponding authors. KAUNS, NM, IF and RA contributed equally and share joint second authorship. SA, ShA, MA, AM, SA, MAl, LK, RK and MG added to the conception, designing and manuscript drafting. SA, ShA and MoA proposed the hypothesis and study design. MA, MuA, SiA, MKA, NM, MG, ZH, MKA, SR, ZH, ZS, contributed biochemical, dosimetry, pharmacological as well as pharmaceutical inputs. SA, MoA, SR, AZ, RK and SR drafted the first version of the manuscript. Doctors Lounge Consortium IF, RA, MSS, SR, AH, AM, ZS, ZA, AK, KH, GAm, MiK, SA, $\mathrm{MH}, \mathrm{QuAl}, \mathrm{AmA}, \mathrm{ABA}, \mathrm{MU}, \mathrm{ABH}, \mathrm{SSHS}, \mathrm{ZS}, \mathrm{AnA}, \mathrm{MK}, \mathrm{TM}$ and $\mathrm{MU}$ contributed significantly to designing the final methodology. MKA, SoA, and AH provided statistical inputs. AAM, Usl, MSu, SZ, SS, SSA, MIA, AmM, $\mathrm{TM}, \mathrm{AH}, \mathrm{YMA}, \mathrm{QAS}, \mathrm{AA}, \mathrm{MoA}$ and $\mathrm{MI}$ have contributed to intellectual inputs in the study protocol and methodology along with final manuscript write up. All authors are responsible for their contributions, providing critical edits and final authorization of the article. The corresponding authors attest trial validity and authenticity.

\section{Acknowledgements}

The authors would like to acknowledge Prof. Dr. Zaheer Ahmad, PhD (Department of botany, Government College University, Lahore, Pakistan). We pay gratitude to late Prof. Dr. Zia Ullah (Internal Medicine, Shaikh Zayed Post-Graduate Medical Complex) who died while serving humanity and combating COVID- 
19. We are particularly grateful for the help of our local collaborators including DOCTORS LOUNGE Consortium.

\section{DOCTORS LOUNGE Consortium}

Arz Muhammad ${ }^{1}$, Kanwal Hayat ${ }^{1}$, Ghazala Amjad ${ }^{1}$, Misbah Kousar ${ }^{1}$, Umair Hafeez ${ }^{1}$, Tayyab Mughal ${ }^{1}$, Tayyaba Muzafar ${ }^{1}$, Sibgha Zulfiqar ${ }^{1}$, Saadia Shahzad Alam¹ ${ }^{1}$, Muhammad Imran Anwar ${ }^{1}$, Amber Malik , Talha Mahmud ${ }^{1}$, Ali Arshad ${ }^{2}$, Khawar Nawaz ${ }^{3}$, Muhammad Ismail Khalid Yousaf ${ }^{4}$

Affiliations: 1, Shaikh Zayed Post-Graduate Medical Complex, Lahore, Pakistan. 2, Kingedward Medical University, Lahore, Pakistan. 3, Sunny Downstate/Kings Country Medical Center, New York, USA. 4. University of Louisville, Kentucky, USA.

\section{Supplementary Files}

This is a list of supplementary files associated with this preprint. Click to download.

- ProtocolHNSCOVIDPKIIProphylaxis.pdf 